\title{
WRITING INTEGERS AS SUMS OF PRODUCTS
}

\author{
CHARLES E. CHACE
}

\begin{abstract}
In this paper we obtain an asymptotic expression for the number of ways of writing an integer $N$ as a sum of $k$ products of $l$ factors, valid for $k \geq 3$ and $l \geq 2$. The proof is an application of the Hardy-Littlewood method, and uses recent results from the divisor problem for arithmetic progressions.
\end{abstract}

Notation. The symbol " $:=$ " indicates that the statement is a definition. The notation $f^{l}(x)$ means $(f(x))^{l}$. The symbol "«" or " $\langle-\rangle$ " means the same thing as "«" or " $O(-)$ " except that the implied "constant" may contain $\varepsilon$ powers of the variables involved; e.g., $\log x \lll 1$. If $E(a)$ is an expression involving $a$, then the expression $E\left(a^{-}\right)$means $\lim _{\varepsilon \rightarrow 0} E(a-\varepsilon)$.

\section{INTRODUCTION}

Let $\nu(N ; k, l)$ denote the number of ways of writing the positive integer $N$ as a sum of $k$ terms, each of which is a product of $l$ factors. In this paper we prove the following

Theorem. Suppose $k \geq 3$ and $l \geq 2$. Then

$$
\nu(N ; k, l)=\mu(N ; k, l)+E(N ; k, l)
$$

where the main terms satisfies

$$
\mu(N ; k, l) \asymp N^{k-1} \log ^{k(l-1)} N,
$$

and the error term satisfies

$$
E(N ; k, l) \lll \begin{cases}N^{k-1-\frac{2(k-2)}{(k-1)(l+1)}} & \text { if } k=3 \text { or } l=2 \text { or } 3 ; \\ N^{k-1-\frac{3(k-2)}{(k-1)(l+2)}} & \text { if } k \text { and } l \geq 4 .\end{cases}
$$

The main term $\mu(N ; k, l)$ is unfortunately rather complicated. It is a sum of terms of the form $\mathscr{S} \mathscr{F}$ where the $\mathscr{S}$ are the "singular series" and the $\mathscr{I}$ are the "singular integrals" which occur in applications of the Hardy-Littlewood method; actually, only the "top" singular series (see §7) and integral deserve the name in the classical sense. The singular integrals satisfy $\mathscr{I} \asymp N^{k-1} \log ^{J} N$ for varying values of $J$; the largest value of $J$ being $k(l-1)$. By imitating the argument used for the singular series in Waring's problem in Chapter 5 of [D], we show that the corresponding singular series is bounded away from zero

Received by the editors October 22, 1993.

1991 Mathematics Subject Classification. Primary 11P55, 11 D85.

Key words and phrases. Additive divisor problem, Hardy-Littlewood method. 
independently of $N$, which shows that the main term is of the order stated in the theorem.

The proof of the theorem is a straightforward application of the HardyLittlewood method (cf. Chapter 3 of [V]). In $\S 2$ we define the major and minor arcs, and in $\S 3$ we obtain a bound for the contribution from the minor arcs. In $\S 4$ we recall some results from the divisor problem for arithmetic progressions, which we use in $\S 5$ to calculate the contribution from the major arcs, isolating the main term and estimating the remainder. In $\S 6$ we define the singular series and integrals and give the order of the latter. Finally, in $\S 7$ we study the "top" singular series and show that the main term is of the required order.

This problem was considered by Estermann in the cases when $k=2$ or 3, and $l=2$. In [E1] he obtained the result $E(N ; 3,2) \lll N^{3 / 2}$ with the main term $\mu(N ; 3,2)$ roughly on the order of $N^{2} \log ^{3} N$, and in [E2], $E(N ; 2,2) \lll$ $N^{7 / 8}$ with $\mu(N ; 2,2)$ roughly on the order of $N \log ^{2} N$. His result is stronger than ours in the case $k=3, l=2$ (ours is $E(N ; 3,2) \lll N^{5 / 3}$ ); his proof uses functional properties of Dirichlet $L$-functions and does not easily extend to the case of larger $k$ and $l$. The most interesting (and difficult) case is when $k=2$ and $l \geq 3$. Here the Hardy-Littlewood method gives a heuristic expression for the main term, since the singular series and integrals converge; as yet, no one has been able to find a smaller bound for the error term. For a discussion of these binary additive divisor problems, see Chapter III of [L].

This paper consists mainly of improvements of results from my doctoral dissertation, and I wish to acknowledge the inestimable help (and inexhaustible patience) of my advisor, P. X. Gallagher. I also wish to thank William Duke and Keith Pardue for helpful suggestions on the material in $\S 7$, and an anonymous referee, whose careful reading and suggestions substantially improved this paper.

\section{DEFINITION OF MAJOR AND MINOR ARCS}

Let $\nu(N ; k, l)$ be the number of ways of writing $N$ as a sum of $k$ products, each containing $l$ factors. Then

$$
\nu(N ; k, l)=\int_{I} S_{l}(\alpha, N)^{k} e(-N \alpha) d \alpha
$$

where $I$ is an interval of unit length and

$$
S_{l}(\alpha, N):=\sum_{n \leq N} d_{l}(n) e(n \alpha) .
$$

We will denote $S_{l}(\alpha, N)$ by $S_{l}(\alpha)$ or just $S(\alpha)$ when the meaning is clear. The major arcs will be short intervals around rational points of small denominator; a typical such arc will have the form

$$
\left[\frac{a}{q}-\frac{1}{q Q}, \frac{a}{q}+\frac{1}{q Q}\right)
$$

where $(a, q)=1$ and $q \leq D$. We shall see later that it is convenient to choose the parameters $D$ and $Q$ such that

$$
D Q=N \text { and } D \leq N^{1 / l} .
$$


If we take $I=\left[-\frac{1}{Q}, 1-\frac{1}{q Q}\right)$ then the union of the major arcs is given by

$$
\mathscr{M}:=\bigcup_{q \leq D} \bigcup_{\substack{a=0 \\(a, q)=1}}^{q-1}\left[\frac{a}{q}-\frac{1}{q Q}, \frac{a}{q}+\frac{1}{q Q}\right)
$$

and the union $\mu$ of minor arcs is just the complement of $\mathscr{M}$ in $I$.

\section{THE INTEGRAL OVER THE MINOR ARCS}

In this section we estimate

$$
\int_{\mu}:=\int_{\mu} S(\alpha)^{k} e(-N \alpha) d(\alpha)
$$

We have

$$
\int_{\mu} \leq \max _{\alpha \in \mu}|S(\alpha)|^{k-2} \int_{0}^{1}|S(\alpha)|^{2} d(\alpha)
$$

and then from the definition (2.1) of $S(\alpha)$ and Parseval's identity we see that

$$
\int_{0}^{1}|S(\alpha)|^{2} d(\alpha) \lll_{l} N,
$$

so it remains to estimate $S(\alpha)$ on $\mu$.

First we show

$$
S(\alpha) \lll_{l} \sum_{n \leq N^{1-1 / l}} \min \left\{\frac{N}{n},\|n \alpha\|^{-1}\right\} .
$$

To prove this we recall that $S(\alpha)=S_{l}(\alpha, N)$ and use induction on $l$. When $l=1$ we see from its definition that

$$
S_{1}(\alpha, N) \ll \min \left\{N,\|\alpha\|^{-1}\right\} .
$$

Now suppose (3.1) is true with $l$ replaced by $l-j$, where $1 \leq j \leq l-1$. We write

$$
S_{l}(\alpha, N)=\sum_{n_{1} \cdots n_{l} \leq N} e\left(n_{1} \cdots n_{l} \alpha\right)
$$

and by an inclusion-exclusion argument this becomes

$$
\begin{aligned}
S_{l}(\alpha, N)= & \sum_{j=1}^{l-1}(-1)^{j-1}\left(\begin{array}{l}
l \\
j
\end{array}\right) \sum_{n_{1} \cdots n_{j} \leq N^{1 / l}} S_{l-j}\left(n_{1} \cdots n_{j} \alpha, \frac{N}{n_{1} \cdots n_{j}}\right) \\
& +(-1)^{l-1} \sum_{n_{1}, \ldots, n_{l} \leq N^{1 / t}} e\left(n_{1} \cdots n_{l} \alpha\right) .
\end{aligned}
$$

By induction we have

$$
\begin{aligned}
& \sum_{n_{1} \cdots n_{j} \leq N^{1 / l}} S_{l-j}\left(n_{1} \cdots n_{j} \alpha, \frac{N}{n_{1} \cdots n_{j}}\right) \\
& \ll_{l} \sum_{n_{1} \cdots n_{j} \leq N^{1 / l}} \sum_{n \leq\left(N /\left(n_{1} \cdots n_{j}\right)\right)^{1-1 /(l-j)}} \min \left\{\frac{N}{n n_{1} \cdots n_{j}},\left\|n n_{1} \cdots n_{j} \alpha\right\|^{-1}\right\}
\end{aligned}
$$


and for the summation conditions $n n_{1} \cdots n_{j} \leq N^{1-1 / l}$ and so

$$
\begin{aligned}
& \sum_{n_{1} \cdots n_{j} \leq N^{1 / l}} S_{l-j}\left(n_{1} \cdots n_{j} \alpha, \frac{N}{n_{1} \cdots n_{j}}\right) \\
& \lll_{l} \sum_{m \leq N^{1-1} / l} d_{j+1}(m) \min \left\{\frac{N}{m},\|m \alpha\|^{-1}\right\} ;
\end{aligned}
$$

here we have replaced the sum on the right-hand side of (3.2) with a larger sum, by setting $m=n n_{1} \cdots n_{j}$ and summing over all ways of writing an integer $m \leq N^{1-1 / l}$ as a product of $j+1$ factors. Similarly we can write

$$
\begin{aligned}
\sum_{n_{1}, \ldots, n_{l} \leq N^{1 / l}} e\left(n_{1} \cdots n_{l} \alpha\right) & =\sum_{n_{1}, \ldots, n_{l-1} \leq N^{1 / l}} S_{1}\left(n_{1} \cdots n_{l-1} \alpha, N^{1 / l}\right) \\
& \lll \sum_{m \leq N^{1-1 / l}} d_{l-1}(m) \min \left\{N^{1 / l},\|m \alpha\|^{-1}\right\} .
\end{aligned}
$$

Note that $d_{j}(m) \ll_{l} 1$ for $j<l$ and that $N^{1 / l} \leq N / m$ when $m \leq N^{1-1 / l}$, so the contribution from (3.4) is dominated by that of (3.3), and (3.1) is proved.

We now require the following

Lemma 3.1. Suppose $|\alpha-a / q|<1 / q^{2}$, where $(a, q)=1$, and let $X$ and $Y \geq 1$. Then

$$
\sum_{n \leq X} \min \left\{X Y n^{-1},\|n \alpha\|^{-1}\right\} \lll X Y\left(q^{-1}+Y^{-1}+q(X Y)^{-1}\right) .
$$

Proof. See Lemma 2.2 of [V].

If $\alpha \in \mu$ then there is a rational number in lowest terms $a / q$ with $D<q \leq Q$ and

$$
\left|\alpha-\frac{a}{q}\right|<\frac{1}{q Q}
$$

by Dirichlet's theorem. We apply Lemma 3.1 with $X=N^{1-1 / l}$ and $Y=N^{1 / l}$ to $(3.1)$ and get

$$
S(\alpha) \ll_{l} N D^{-1}+N^{1-1 / l}+Q .
$$

Using the conditions (2.2) on $D$ and $Q$, we then have

$$
\int_{\mu} \ll_{l} N^{k-1} D^{-k+2} \text {. }
$$

\section{THE DIVISOR PROBLEM OVER ARITHMETIC PROGRESSIONS}

For $\alpha \in \mathscr{M}$, we write $\alpha=a / q+\beta$ with $(a, q)=1, q \leq D$ and $|\beta| \leq$ $(q Q)^{-1}$. Then we write

$$
S(\alpha)=\sum_{b=1}^{q} e\left(\frac{a b}{q}\right) \sum_{\substack{n \leq N \\ n \equiv b \bmod q}} d_{l}(n) e(n \beta) .
$$

The inner sum can be expressed as a Stieltjes integral

$$
\int_{1^{-}}^{N} e(\beta x) d D_{l}(x ; b, q)
$$


where

$$
D_{l}(x ; b, q):=\sum_{\substack{n \leq N \\ n \equiv b \bmod q}} d_{l}(n) .
$$

We now make use of a "main term plus remainder" expression for this sum. The main term has the form

$$
M_{l}(x ; b, q):=\sum_{j=0}^{l-1} c_{j+1}(b, q) L_{j}(x)
$$

for some coefficients $c_{j}(b, q)$ given explicitly in equation (2.13) of [C]; here $L_{j}(x)$ is an antiderivative of $\log ^{j} x / j !$. The error term has an upper bound of the form

$$
\Delta_{l}(x ; b, q) \lll_{l}\left(\frac{x}{g}\right)^{\eta}
$$

provided $q \leq x^{1 / l} ;$ here $g=(b, q)$. The values for $\eta$ which we will use are

$$
\eta=\frac{l-1}{l+1}
$$

a result of $\mathrm{K}$. Matsumoto [M] valid for $g=1$, to which we apply a technique of Heath-Brown from $\S 8$ of $[\mathrm{H}]$ for removing the restriction on $g$, and

$$
\eta=\frac{l-1}{l+2}
$$

provided $l \geq 4$; see Theorem 2 of $[\mathrm{C}]$.

To figure the contribution from $\Delta_{l}(x ; b, q)$ to $S(\alpha)$ we integrate by parts and estimate

$$
E:=\int_{1^{-}}^{N} e(\beta x) d \Delta_{l}(x ; b, q) \lll_{l}(1+N|\beta|) \max _{l^{-}<x \leq N}\left|\Delta_{l}(x ; b, q)\right| .
$$

Suppose $q \leq N^{1 / l}$. Then for $x \geq q^{l}$ we apply the estimate above, and for $x<q^{l}$ we apply the elementary estimate

$$
\Delta_{l}(x ; b, q) \lll_{l} x^{1-1 / l}+q^{l-1}
$$

to obtain

$$
E \lll_{l}(1+N|\beta|)\left(q^{l-1}+\frac{N^{\eta}}{g}\right) .
$$

Summing over $b$ and assuming $D \leq N^{1 / l}$ we see that the contribution to $S(\alpha)$ is

$$
\left\langle D^{l}+N^{\eta} D\right\rangle \text {. }
$$

The contribution of $M_{l}(x ; b, q)$ to $S(\alpha)$ can be written as

$$
\sum_{j=0}^{l-1} A_{j}(q) I_{j}(\beta)
$$

where

$$
A_{j}(q):=\sum_{b=1}^{q} e\left(\frac{a b}{q}\right) c_{j}(b, q)
$$


and

$$
I_{j}(\beta):=\int_{1}^{N} e(\beta x) \frac{\log ^{j} x}{j !} d x .
$$

For $(a, q)=1, A_{j}(q)$ is independent of $a$. The coefficients $c_{j}(b, q)$ are sums of terms of the form

$$
\sum_{b_{1} b_{2} \equiv b \bmod q} f\left(b_{1}\right)
$$

for some function $f$; the number of terms in the sum depends only on $l$, and we have

$$
\sum_{b=1}^{q} e\left(\frac{a b}{q}\right) \sum_{b_{1} b_{2} \equiv b \bmod q} f\left(b_{1}\right)=\sum_{b_{1}=1}^{q} f\left(b_{1}\right) \sum_{b_{2}=1}^{q} e\left(\frac{a b_{1} b_{2}}{q}\right) .
$$

Since $(a, q)=1$ the inner sum vanishes unless $b_{1}=q$, and so $A_{j}(q)$ is a sum of terms of the form $q f(q)$.

We use this to estimate $A_{j}(q)$. From equation (2.13) of [C] we see that $f$ has the form

$$
f(a):=\frac{1}{q^{i}} \sum_{a_{1} \cdots a_{l} \equiv a \bmod q} \gamma_{m_{i}}\left(a_{i}, q\right) \cdots \gamma_{m_{l}}\left(a_{l}, q\right)
$$

where $1 \leq i \leq l$, and the $\gamma_{m}(a, q)$ are Laurent coefficients of the Hurwitz zeta function

$$
\sum_{\substack{n \geq 1 \\ n \equiv a \bmod q}} \frac{1}{n^{s}}
$$

Using an expression analogous to one for the Laurent coefficients of the Riemann zeta function, it is not difficult to show that $\gamma_{m}(a, q) \lll a^{-1}$. Elementary congruence arguments then give the bound

$$
A_{j}(q) \lll_{l} q^{-1}
$$

for full details, see $\S 3$ and $\S 4$ of $[C]$. that

Finally we estimate $I_{j}(\beta)$ trivially, and then after integrating by parts to see

$$
I_{j}(\beta) \lll_{l} \min \left\{N,|\beta|^{-1}\right\}
$$

\section{THE INTEGRAL OVER THE MAJOR ARCS}

We now consider the integral

$$
\int_{\mathscr{M}}:=\int_{\mathscr{M}} S(\alpha)^{k} e(-N \alpha) d \alpha
$$

From the preceding section, we can write $S(\alpha)=\Sigma+R$, where $\Sigma$ is given by (4.5) and $R$ is bounded by (4.4). Hence

$$
S(\alpha)^{k}=\Sigma^{k}+O\left(R\left[S(\alpha)^{k-1}+\Sigma^{k-1}\right]\right) .
$$

Now

$$
\int_{\mathscr{M}}|S(\alpha)|^{k-1} d \alpha \lll_{l} N^{k-2}
$$


by the same argument used in $\S 3$, and the fact that $S(\alpha) \ll_{l} N$. One then shows that

$$
\int_{\mathscr{M}}|\Sigma|^{k-1} d \alpha \lll_{l} N^{k-2}
$$

using the definition of major arcs, the assumption (2.2) on $D$ and $Q$, and the bounds (4.8) and (4.9) on $A_{j}(q)$ and $I_{j}(\beta)$. Hence

$$
\int_{\mathscr{M}}=\int_{\mathscr{M}} \Sigma^{k} e(-N \alpha) d \alpha+\left\langle R N^{k-2}\right\rangle .
$$

Consider the integral on the right-hand side. From the definitions of $\mathscr{M}$ and $\Sigma$ this is

where

$$
\sum_{q \leq D} C_{q}(N) \int_{-1 / q Q}^{1 / q Q}\left[\sum_{j=0}^{l-1} A_{j}(q) I_{j}(\beta)\right]^{k} e(-N \beta) d \beta
$$

$$
C_{q}(N):=\sum_{a=1}^{q} e\left(\frac{-N a}{q}\right)
$$

is Ramanujan's sum. We now extend the sum to all $q$ and the integral to all $\beta$. This effectively allows us to disentangle the "arithmetic" part, i.e. expressions involving $q$, from the "continuous" part, involving $\beta$. The former will become the singular series, the latter, the singular integrals of the problem. Convergence is assured by the bounds (4.8) and (4.9), provided $k \geq 3$. These bounds are also used to estimate the error introduced. The error from extending the integral to all $\beta$ is on the order of

$$
\sum_{q \leq D} q \int_{1 / q Q}^{\infty}\left|\sum_{j=0}^{l-1} A_{j}(q) I_{j}(\beta)\right|^{k} d \beta
$$

from extending the sum, it is on the order of

$$
\sum_{q>D} q \int_{-\infty}^{\infty}\left|\sum_{j=0}^{l-1} A_{j}(q) I_{j}(\beta)\right|^{k} d \beta
$$

Estimating and using (2.2), both of these are $\left\langle N^{k-1} D^{-k+2}\right\rangle$, which is the same as the bound (3.5) on the integral over the minor arcs. Hence we have

$$
\nu(N ; k, l)=\mu(N ; k, l)+E(N ; k, l)
$$

where the main term is given by

$$
\mu(N ; k, l):=\sum_{q=1}^{\infty} C_{q}(N) \int_{-\infty}^{\infty}\left[\sum_{j=0}^{l-1} A_{j}(q) I_{j}(\beta)\right]^{k} e(-N \beta) d \beta,
$$

and from (5.1) and (4.4) the error term has the bound

$$
E(N ; k, l) \lll N^{k-2}\left(N D^{-k+2}+D^{l}+N^{\eta} D\right) .
$$

Taking $D=N^{\delta}$ where

$$
\delta=\min \left\{\frac{1}{k+l-2}, \frac{1-\eta}{k-1}\right\}
$$


we obtain

$$
E(N ; k, l) \lll_{k, l} N^{k-2}\left(N^{\frac{1}{k+l-2}}+N^{\frac{\eta(k-2)+1}{k-1}}\right)
$$

and the restriction $\delta \leq 1 / l$ is more than satisfied for $k \geq 3$. Using the values (4.2) and (4.3) for $\eta$ give the bound stated in the theorem.

\section{SINGULAR SERIES AND INTEGRALS}

Expanding the integrand in (5.2) we can write

$$
\mu(N ; k, l)=\sum_{\substack{\mathbf{j}=\left(j_{1}, \ldots, j_{k}\right) \\ 0 \leq j_{1}, \ldots, j_{k} \leq l-1}} \mathscr{S}_{\mathbf{j}} \mathscr{Y}_{\mathbf{j}}
$$

where a typical singular series is given by

$$
\mathscr{S}_{\mathbf{j}} \equiv \sum_{q=1}^{\infty} C_{q}(N) \prod_{i=1}^{k} A_{j_{i}}(q)
$$

(absolute convergence being assured by the estimate (4.8) for $A_{j}(q)$ ), and the corresponding singular integral is

$$
\mathscr{Y}_{\mathbf{j}}:=\int_{-\infty}^{\infty} \prod_{i=1}^{k} I_{j_{i}}(\beta) e(-N \beta) d \beta
$$

We can simplify this last expression considerably. In the definition (4.7) of $I_{j}$ write

$$
l_{j}(x)=\frac{\log ^{j} x}{j !}
$$

for convenience. If we set

$$
\mathscr{L}(x)=\frac{d}{d x}\left(\int_{\substack{x_{1}+\cdots+x_{k} \leq x \\ \leq x_{1}, \ldots, x_{k} \leq N}} \ldots \prod_{i=1}^{k} l_{j_{i}}\left(x_{i}\right) d x_{1} \cdots d x_{k}\right)
$$

then

$$
\prod_{i=1}^{k} I_{j_{i}}(\beta)=\int_{-\infty}^{\infty} e(\beta x) \mathscr{L}(x) d x
$$

and so $\mathscr{Y}_{\mathrm{j}}=\mathscr{L}(N)$ by Fourier inversion. The following lemma provides a neater expression for $\mathscr{L}$.

Lemma 6.1. Let $k \geq 2$ be an integer and let

$$
f(x)=\int_{\substack{x_{1}+\cdots+x_{k} \leq x \\ x_{1}, \ldots, x_{k} \geq 0}} \phi\left(x_{1}, \ldots, x_{k}\right) d x_{1} \cdots d x_{k}
$$

for some continuous function $\phi$. Then

$$
f^{\prime}(x)=\int_{\substack{x_{1}+\cdots+x_{k-1} \leq x \\ x_{1}, \ldots, x_{k-1} \geq 0}} \phi\left(x_{1}, \ldots, x_{k-1}, x-\left(x_{1}+\cdots+x_{k-1}\right)\right) d x_{1} \cdots d x_{k-1} .
$$


Proof. We first prove the case $k=2$, and then induct on $k$. Let

$$
f(x)=\iint_{\substack{x_{1}+x_{2} \leq x \\ x_{1}, x_{2} \geq 0}} \phi\left(x_{1}, x_{2}\right) d x_{1} d x_{2}=\int_{0}^{x} \Phi\left(x, x_{1}\right) d x_{1}
$$

where

$$
\Phi\left(x, x_{1}\right):=\int_{0}^{x-x_{1}} \phi\left(x_{1}, x_{2}\right) d x_{2} .
$$

We use the fact that

$$
\frac{d}{d x} \int_{a}^{x} g(x, y) d y=g(x, x)+\int_{a}^{x} \frac{\partial}{\partial x} g(x, y) d y
$$

with $g=\Phi$, and note that $\Phi(x, x)=0$ and that

$$
\frac{\partial}{\partial x} \Phi\left(x, x_{1}\right)=\phi\left(x_{1}, x-x_{1}\right) \text {, }
$$

So

$$
f^{\prime}(x)=\int_{0}^{x} \phi\left(x_{1}, x-x_{1}\right) d x_{1}
$$

which is the lemma when $k=2$.

Now let

$$
\begin{aligned}
f(x) & =\int_{\substack{x_{1}+\cdots+x_{k} \leq x \\
x_{1}, \ldots, x_{k} \geq 0}} \phi\left(x_{1}, \ldots, x_{k}\right) d x_{1} \cdots d x_{k} \\
& =\int_{0}^{x} \Phi\left(x, x_{1}\right) d x_{1}
\end{aligned}
$$

where

$$
\Phi\left(x, x_{1}\right)=\int_{\substack{x_{2}+\cdots+x_{k} \leq x-x_{1} \\ x_{2}, \ldots, x_{k} \geq 0}} \phi\left(x_{1}, x_{2}, \ldots, x_{k}\right) d x_{2}, \ldots, d x_{k} .
$$

As before we have

$$
f^{\prime}(x)=\Phi(x, x)+\int_{0}^{x} \frac{\partial}{\partial x} \Phi\left(x, x_{1}\right) d x_{1}
$$

and $\Phi(x, x)=0$. Then by induction,

$$
\begin{aligned}
& \frac{\partial}{\partial x} \Phi\left(x, x_{1}\right) \\
& =\int_{\substack{x_{2}+\cdots+x_{k-1} \leq x-x_{1} \\
x_{2}, \ldots, x_{k-1} \geq 0}} \phi\left(x_{1}, x_{2}, \ldots, x_{k-1},\left(x-x_{1}\right)-\left(x_{2}+\cdots+x_{k-1}\right)\right) d x_{2} \cdots d x_{k-1}
\end{aligned}
$$

and the lemma is proved.

Applying the lemma we have

$$
\mathscr{F}_{\mathbf{j}}=\int_{\substack{x_{1}+\cdots+x_{k-1} \leq N-k+2 \\ x_{1}, \ldots, x_{k-1} \geq 1}}\left(\prod_{i=1}^{k-1} l_{j_{i}}\left(x_{i}\right)\right) l_{j_{k}}\left(N-\sum_{i=1}^{k-1} x_{i}\right) d x_{1} \cdots d x_{k-1}
$$


from which we see that

$$
\mathscr{Y}_{\mathbf{j}} \asymp_{k, l} N^{k-1} \log ^{J} N
$$

where $J=\sum_{i=1}^{k} j_{i}$. Since all of the singular series can be bounded above independently of $N$, the order of the "top" singular integral, where all $j_{i}=l-1$, will be the order of the whole main term $\mu(N ; k, l)$ if the "top" singular series is also bounded below independently of $N$.

\section{THE "TOP" SINGULAR SERIES}

The top singular series is $\mathscr{S}=\mathscr{S}_{\mathbf{j}}$ where $j_{i}=l-1$ for all $i$, i.e.

$$
\mathscr{S}=\sum_{q=1}^{\infty} C_{q}(N) A_{l-1}(q)^{k} .
$$

To show that the main term has the order stated in the theorem, we must show that this is bounded away from zero independently of $N$. The argument is much like that for the singular series for Waring's problem, given in Chapter 5 of [D]. The idea is to write the series as an Euler product and to estimate the factors in two different ways. The first is a straightforward estimate which shows that the product over large primes is bounded away from zero. The second involves interpreting the factors as local densities of solutions to the polynomial equation which describes writing $N$ as a sum of products, and then showing that there are enough solutions to bound the factors away from zero.

From the definition of $A_{j}(q)$ given in (4.6) and the fact that

$$
c_{l-1}(a, q)=\frac{1}{q^{l}} \sum_{a_{1} \cdots a_{l-1} \equiv a \bmod q} 1
$$

where the sum is taken over $a_{1}, \ldots, a_{l-1} \bmod q$ (see (4.1), and equation (2.13) of [C]), the argument which shows that $A_{j}(q)$ is independent of $a$ shows that

$$
A_{l-1}(q)=\frac{1}{q^{l-1}} \sum_{a_{1} \cdots a_{l-1} \equiv 0 \bmod q} 1 .
$$

If we write

$$
s_{j}(q):=q \sum_{a_{1} \cdots a_{j} \equiv 0 \bmod q} 1
$$

then

$$
\mathscr{S}=\sum_{q=1}^{\infty} T(q)
$$

where

$$
T(q):=C_{q}(N) s_{l-1}(q)^{k} q^{-k l} .
$$

In order to write $\mathscr{S}$ as an Euler product and estimate the factors, we must show that $T(q)$ is multiplicative and obtain an estimate for it. We require the following 
Lemma 7.1. For $s_{j}(q)$ defined as in (7.1) we have $s_{1}(q)=q$, and for $j>1$

$$
s_{j}(q)=\sum_{d \mid q} \phi(d)\left(\frac{q}{d}\right)^{j} s_{j-1}(d) .
$$

Proof. We have

$$
s_{j}(q):=q \sum_{a_{j}=1}^{q} \sum_{\substack{1 \leq a_{1}, \ldots, a_{j-1} \leq q \\ q \mid a_{1} \cdots a_{j}}} 1 .
$$

We then write $a_{j}=c q / d$ where $q / d=\left(a_{j}, q\right)$. The condition $q \mid a_{1} \cdots a_{j}$ is then equivalent to $d \mid a_{1} \cdots a_{j-1}$. The sum over $a_{i} \bmod q$ can then be written as $q / d$ copies of a sum over $a_{i} \bmod d$. Hence

$$
s_{j}(q) \equiv q \sum_{d \mid q} \sum_{\substack{c=1 \\
(c, d)=1}}^{d}\left(\frac{q}{d}\right)^{j-1} \sum_{\begin{array}{r}
1 \leq a_{1}, \ldots, a_{j-1} \leq d \\
d \mid a_{1} \cdots a_{j-1}
\end{array}} 1
$$

which gives the stated result.

Since the lemma is a recursive statement, we can prove easily by induction that $s_{j}(q)$ is multiplicative in $q$, and that $q^{j} \ll s_{j}(q) \lll q^{j}$. Using this, and multiplicativity and estimates for Ramanujan's sum $C_{q}(N)$, we see that $T(q)$ is indeed multiplicative and that $T(q) \lll q^{-k+1}$ independently of $N$. Hence we can write

$$
\mathscr{S}=\prod_{p} F(p)
$$

where

$$
F(p)=\sum_{\nu=0}^{\infty} T\left(p^{\nu}\right) .
$$

By the preceding bound on $T(q)$ and the fact that $k \geq 3$ we have $|F(p)-1| \lll$ $p^{-k+1}$ and so we can find a prime $p_{0}$ such that

$$
\frac{1}{2}<\prod_{p>p_{0}} F(p)<\frac{3}{2}
$$

Taking a more general view, let $f$ be a polynomial of degree $D$ in $m$ variables, with integer coefficients. If we consider the "generic" additive problem of counting solutions $\mathbf{x}$ in $\mathbf{Z}^{m}$ (or some subset) to the equation $f(\mathbf{x})=N$, then we define the singular series as follows:

$$
\mathscr{S}:=\sum_{q=1}^{\infty} \frac{1}{q^{m}} \sum_{\substack{r=1 \\(r, q)=1}}^{q} e\left(-\frac{r n}{q}\right) \sum_{\mathbf{x m o d} q} e\left(\frac{r}{q} f(\mathbf{x})\right),
$$

where the innermost sum is over all $\mathbf{x}$ in $(\mathbf{Z} / q \mathbf{Z})^{m}$. This corresponds with the singular series defined above for our particular problem. By a straightforward generalization of the proofs of Lemmas 6, 7, and 8 of [D], one can show that the factors $F(p)$ in the Euler product for $\mathscr{S}$ can be written

$$
F(p)=\lim _{\nu \rightarrow \infty} \frac{M\left(p^{\nu}, f, N\right)}{p^{\nu(m-1)}}
$$


where $M(q, f, N)$ is the number of $\mathbf{x}$ in $(\mathbf{Z} / q \mathbf{Z})^{m}$ with $f(\mathbf{x}) \equiv N \bmod q$. We can thus obtain lower bounds on $F(p)$ by showing that there are sufficiently many solutions to the polynomial congruence.

Lemma 7.2. Let $f$ be a polynomial of degree $D$ in $m$ variables, with integer coefficients. Suppose there is some a in $\left(\mathbf{Z} / p^{\nu} \mathbf{Z}\right)^{m}$ such that $f(\mathbf{a}) \equiv N \bmod p^{\nu}$ and the gradient vector $\nabla f(\mathbf{a})$ is not zero $\bmod p$. Then there are at least $p^{m-1}$ distinct elements $\mathbf{b}$ of $\left(\mathbf{Z} / p^{\nu+1} \mathbf{Z}\right)^{m}$ which satisfy $f(\mathbf{b}) \equiv N \bmod p^{\nu+1}$.

Proof. Let $\mathbf{b}=\mathbf{a}+p^{\nu} \mathbf{y}$, where $\mathbf{y}$ is in $(\mathbf{Z} / p \mathbf{Z})^{m}$. Then

$$
f(\mathbf{b}) \equiv f(\mathbf{a})+p^{\nu}(\nabla f(\mathbf{a}) \cdot \mathbf{y}) \bmod p^{\nu+1} .
$$

Since $f(\mathbf{a})=N+c p^{\nu}$ for some integer $c$, every $\mathbf{y}$ for which

$$
\nabla f(\mathbf{a}) \cdot \mathbf{y} \equiv-c \bmod
$$

gives a vector $\mathbf{b}$ which satisfies the required congruence. If $\nabla f(\mathbf{a})$ is not zero $\bmod p$, there are $p^{m-1}$ choices for $\mathbf{y}$.

Returning to our particular case, let

$$
f(\mathbf{x})=\sum_{i=1}^{k} \prod_{j=1}^{l} x_{i j} .
$$

If $N$ is sufficiently large (for example, bigger than $k$ ), let $\mathbf{a}=(1,1, \ldots, 1, N-$ $k+1)$. Then $f(\mathbf{a})=N$, and we can use the lemma to construct solutions to the congruence $f(\mathbf{x}) \equiv N \bmod p^{\nu}$. The gradient condition is fulfilled since $\nabla f(\mathbf{a})$ is not zero $\bmod p$ for any prime $p$, and from the construction in the proof of the lemma, we see that all solutions are congruent component-wise to a $\bmod p$. Distinctness of solutions is also guaranteed by the construction, so we have $M\left(p^{\nu}, f, N\right) \geq p^{(\nu-1)(k l-1)}$, and so $F(p) \geq 1 / p^{k l-1}$. This and (7.4) give what we want, namely a positive lower bound for $\mathscr{S}$ independent of $N$.

We may also obtain a more explicit expression for the factors $F(p)$. First, we use Lemma 7.1 to derive an expression for $s_{j}$ at primes powers:

$$
s_{j}\left(p^{\alpha}\right)=p^{\alpha j} \sum_{i=0}^{j-1}\left(\begin{array}{c}
\alpha-1+i \\
i
\end{array}\right)\left(1-\frac{1}{p}\right)^{i}
$$

for $\alpha \geq 1$. We then compute $F(p)$ using (7.2), (7.3), and Theorem 272 of [HW], which implies that

$$
C_{p^{\alpha}}(N)= \begin{cases}\phi\left(p^{\alpha}\right) & \text { if } \alpha \leq \nu, \\ -p^{\nu} & \text { if } \alpha=\nu+1, \\ 0 & \text { if } \alpha>\nu+1,\end{cases}
$$

where $N=\prod p^{n}$. We distinguish two cases: $p \nmid N$ and $p \mid N$.

If $p \nmid N$, then the local factor is

$$
F(p)=1-\left(1-\left(1-\frac{1}{p}\right)^{l-1}\right)^{k}
$$

since the sum in (7.5) is just a partial sum of a geometric series when $\alpha=1$. 
When $p \mid N$, the local factor is much more complicated:

$$
\begin{aligned}
F(p)= & 1+\sum_{j=1}^{\nu} p^{-j(k-1)} y\left[\sum_{i=0}^{l-2}\left(\begin{array}{c}
j-1+i \\
i
\end{array}\right) y^{i}\right]^{k} \\
& -p^{-(\nu+1)(k-1)-1}\left[\sum_{i=0}^{l-2}\left(\begin{array}{c}
\nu+i \\
i
\end{array}\right) y^{i}\right]^{k}
\end{aligned}
$$

where for convenience we write $y=1-1 / p$.

\section{BIBLIOGRAPHY}

[C] C. E. Chace, The divisor problem for arithmetic progressions with small modulus, Acta Arith. 61 (1992), 35-50.

[D] H. Davenport, Analytic methods for Diophantine equations and Diophantine inequalities, Campus Publishers, Univ. of Michigan, Ann Arbor, Michigan, 1962.

[E1] T. Estermann, On the representations of a number as the sums of three products, Proc. London Math. Soc. (2) 29 (1929), 453-478.

[E2] - On the representations of a number as the sum of two products, Proc. London Math. Soc. (2) 31 (1930), 123-133.

[HW] G. H. Hardy and E. M. Wright, An introduction to the theory of numbers, 5 th ed., Clarendon Press, Oxford, 1984.

[H] D. R. Heath-Brown, The divisor function $d_{3}(n)$ in arithmetic progressions, Acta Arith. 47 (1987), 29-56.

[L] Ju. V. Linnik, The dispersion method in binary additive problems, Transl. Math. Monos., vol. 4, Amer. Math. Soc., Providence, R.I., 1983.

[M] K. Matsumoto, A remark on Smith's results on a divisor, Nagoya Math. J. 98 (1985), 37-42.

[T] E. C. Titchmarsh, The theory of the Riemann zeta-function, 2nd ed. revised by D. R. HeathBrown, Clarendon Press, Oxford, 1986.

[V] R. C. Vaughan, The Hardy-Littlewood method, Cambridge Tracts in Math., vol. 80, Cambridge Univ. Press, 1981.

Department of Mathematics, Williams College, Williamstown, Massachusetts 01267 Current address: 335 W 21st Street, Apartment FW, New York, New York 10011

E-mail address: charles.e.chace@illiams.edu 